\title{
Disorder-induced mechanism for positive exchange bias fields
}

\author{
Orlando V. Billoni, Francisco A. Tamarit, and Sergio A. Cannas \\ Facultad de Matemática, Astronomía y Física, Universidad Nacional de Córdoba and Instituto de Física Enrique Gaviola (IFEG-CONICET), \\ Ciudad Universitaria, 5000 Córdoba, Argentina \\ (Received 11 April 2013; published 18 July 2013)
}

\begin{abstract}
We propose a mechanism to explain the phenomenon of positive exchange bias on magnetic bilayered systems. The mechanism is based on the formation of a domain wall at a disordered interface during field cooling, which induces a symmetry breaking of the antiferromagnet, without relying on any ad hoc assumption about the coupling between the ferromagnetic (FM) and antiferromagnetic (AFM) layers. The domain wall is a result of the disorder at the interface between FM and AFM, which reduces the effective anisotropy in the region. We show that the proposed mechanism explains several known experimental facts within a single theoretical framework. This result is supported by Monte Carlo simulations on a microscopic Heisenberg model, by micromagnetic calculations at zero temperature, and by mean-field analysis of an effective Ising-like phenomenological model.
\end{abstract}

The exchange bias phenomenon ${ }^{1}$ (EB) usually appears in heterogeneous magnetic systems in the nanoscale range, such as thin-film layered systems. EB has captured the attention of many researchers due to its applications, ${ }^{2,3}$ which make the area an active field of research. ${ }^{4-6}$ For instance, EB is currently applied in the design of spin valves. ${ }^{2,7}$ The phenomenon manifests itself when the system is cooled down in the presence of a magnetic field, provided the starting temperature is above a certain threshold and the final temperature is low enough. A hysteresis loop performed after this procedure shows a horizontal shift called the bias field, $H_{\mathrm{EB}}$. Usually the bias field is opposite to the cooling field [normal exchange bias (NEB)], but sometimes the displacement is in the same direction and it is called positive $\mathrm{EB}^{8}$ (PEB). Other important effects can appear, such as a vertical shift in the magnetization ${ }^{9}$ and the widening and symmetry loss of the hysteresis loops. EB disappears if the system is heated above the blocking temperature, $T_{B}$, which is below but close to the Néel temperature of the antiferromagnetic (AFM).

Currently, much of the effort is focused on tuning EB and establishing the mechanisms which control the effect. The existence of uncompensated domains at the interface has been shown to be fundamental for the appearance of EB. ${ }^{10}$ Also, at the relevant scales of the problem all the systems have some unavoidable amount of disorder which seems to play a main role. In this regard, several routes are employed in experiments to introduce and control the disorder effects. ${ }^{8,11-15}$ For instance, dilution can enhance the bias field. ${ }^{12}$ In addition, the interfacial roughness and the disorder in the anisotropy are related to the appearance of PEB. ${ }^{8,13}$ In any case, it is well established that a strong cooling field is necessary for the observation of PEB. ${ }^{8,9,16}$ Among the bilayered systems, one of the most studied is the $\mathrm{FM} / \mathrm{FeF}_{2}$, because the $\mathrm{AFM} \mathrm{FeF}$ has a simple spin structure. ${ }^{17}$ In particular, PEB was reported for the first time by Nogués et al. ${ }^{8}$ in this kind of system.

Most of the theoretical work up to now has assumed that the AFM/FM interface exchange interaction is antiferromagnetic; this is a key ingredient to explain PEB. ${ }^{18-24}$ In this paper, we show that such an ad hoc assumption (hard to justify physically) is not necessary to explain PEB, as long as a large enough amount of disorder is present at the interface. To exhibit the mechanism behind such an effect, we first performed Monte Carlo simulations using a microscopy model for the bilayered system. We show that PEB is related to the formation of a domain wall at the interface during field cooling (FC), hence in this case PEB is independent of the sign of the interface exchange interaction.

We considered a FM film mounted over an AFM film. The films are magnetically coupled to each other by exchange interactions and the structure of both films is bcc, assuming a perfect match across the FM/AFM interface. The system is ruled by the following Hamiltonian:

$$
\begin{aligned}
H= & -J_{F} \sum_{\left\langle\vec{r}, \vec{r}^{\prime}\right\rangle \in \mathrm{FM}} \vec{S}_{\vec{r}} \cdot \vec{S}_{\vec{r}^{\prime}}-K_{F} \sum_{\vec{r} \in \mathrm{FM}}\left(S_{\vec{r}}^{z}\right)^{2} \\
& +J_{A} \sum_{\left\langle\vec{r}, \vec{r}^{\prime}\right\rangle \in \mathrm{AFM}} \vec{S}_{\vec{r}} \cdot \vec{S}_{\vec{r}^{\prime}}-K_{A} \sum_{\vec{r} \in \mathrm{AFM}}\left(\vec{S}_{\vec{r}} \cdot \hat{n}_{\vec{r}}\right)^{2} \\
& -J_{\mathrm{EB}} \sum_{\left\langle\vec{r}, \vec{r}^{\prime}\right\rangle \in \mathrm{FM} / \mathrm{AFM}} \vec{S}_{\vec{r}} \cdot \vec{S}_{\vec{r}^{\prime}}-h \sum_{\vec{r}} S_{\vec{r}}^{y},
\end{aligned}
$$

where $\vec{S}_{\vec{r}}$ is a classical Heisenberg spin $\left(\left|\vec{S}_{\vec{r}}\right|=1\right)$ located at the node $\vec{r}$ of the lattice. $\left\langle\vec{r}, \vec{r}^{\prime}\right\rangle$ denotes a sum over nearestneighbor pairs of spins. $J_{F}>0$ is the exchange constant of the $\mathrm{FM}, J_{A}>0$ is the strength of the AFM exchange, and $J_{\mathrm{EB}}>0$ is the exchange coupling between the FM and the AFM at the interface. $K_{F}$ and $K_{A}$ are FM and AFM anisotropy constants, respectively. The disorder in the anisotropy is introduced as in the random anisotropy model, ${ }^{25}$ i.e., $\hat{n}_{\vec{r}}$ is a random direction versor for AFM spins close to the interface. Inside the AFM, $\hat{n}_{\vec{r}}$ points in the $y$ direction. $h$ is an external homogeneous magnetic field oriented along the $y$ direction. We assumed $K_{F}<0$ - planar anisotropy-to ensure the FM spins remain in the film plane, mimicking the dipolar shape anisotropy. ${ }^{26}$

Monte Carlo simulations were performed using the METROPOLIS algorithm. $L_{x}=L_{y}=L$ are the lateral dimensions of the films, and $L_{z a}$ and $L_{z f}$ are the number of atomic layer of the FM and AFM films, respectively. We set $L_{x}=L_{y}=20,40, L_{z a}=24$, and $L_{z f}=12$. Periodic boundary conditions were imposed in the plane of the film while open boundary conditions were used in the perpendicular direction. For each point in the magnetization curve, we took 


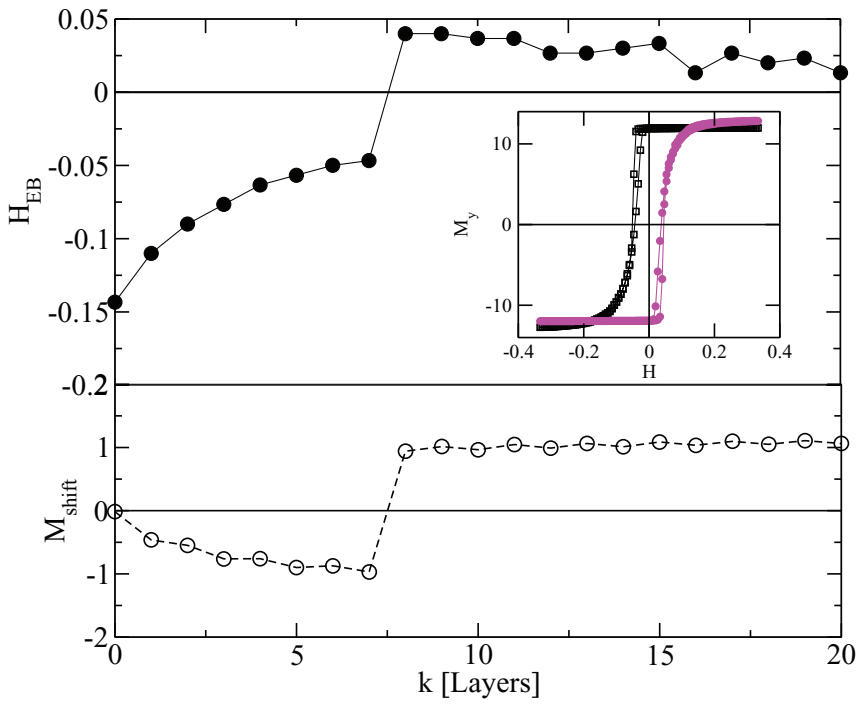

FIG. 1. (Color online) $H_{\mathrm{EB}}$ and $M_{\text {shift }}$ vs the number of disordered planes at low temperature $\left(T / T_{N} \sim 0.1\right.$ and $\left.H_{\mathrm{CF}}=0.32\right)$, top and bottom panel, respectively. Inset: hysteresis loops before and after the change of sign of $H_{\mathrm{EB}}$. Note that $M_{\text {shift }}$ stabilizes to a value close to 1 . This is expected according to Eq. (5).

$10^{4}$ Monte Carlo steps per site (MCS) to thermalize the system and the same number of MCS to calculate temporal averages. The AFM was modeled using $\mathrm{FeF}_{2}$ fluoride parameters ${ }^{27}$ setting AFM interface spin configuration uncompensated, ${ }^{28-30}$ corresponding to the (100) $\mathrm{FeF}_{2}$ crystalline orientation.

In Fig. 1, we show the field $H_{\mathrm{EB}}$ versus the number of disordered AFM layers $k$ in the interface region. The temperature of the system is well below the Néel temperature $\left(T / T_{N}=0.1\right)$. As a general rule, the bias field decreases in module as the number of planes with disorder increases, as expected according to previous results (see, e.g., Ref. 31). The bias field is normal up to $k=7$, and for larger values it becomes positive. Notice that the absolute value of $H_{\mathrm{EB}}$ varies continuously at the transition from normal to PEB, as observed experimentally. ${ }^{8}$ We also observe a vertical shift in the hysteresis loops correlated with the sign of the bias field (see Fig. 1), as observed in fluoride iron compounds. ${ }^{9}$ In Fig. 2 , we plot $H_{\mathrm{EB}}$ versus temperature for a system with a fixed number of AFM disordered layers $(k=12)$ and for different cooling fields. If the cooling field is strong $\left(H_{\mathrm{CF}}>0.24 J_{A}\right)$, $H_{\mathrm{EB}}$ is positive in the whole range of temperatures, whereas as $H_{\mathrm{CF}}$ decreases, the sign of $H_{\mathrm{EB}}$ changes twice at intermediate temperatures. A change of sign of $H_{\mathrm{EB}}$ as a function of the temperature has been observed in diluted $\mathrm{AFM}^{11}$ and in random anisotropy AFM. ${ }^{13}$ As we will discuss later, while NEB is expected at low temperatures, the presence of PEB at very low temperatures appears to be a spurious finite-size effect, as suggested by the strong enhancement of fluctuations in the sign of $H_{\mathrm{EB}}$ as the lateral size of the system is reduced (see the inset of Fig. 2).

An inspection of the local magnetization at each layer shows that, in the case of PEB, an antiferromagnetic domain wall (DW) forms during FC in the disordered region. In this way, the system reduces the exchange energy cost due to frustration while it stores energy at the interface through the

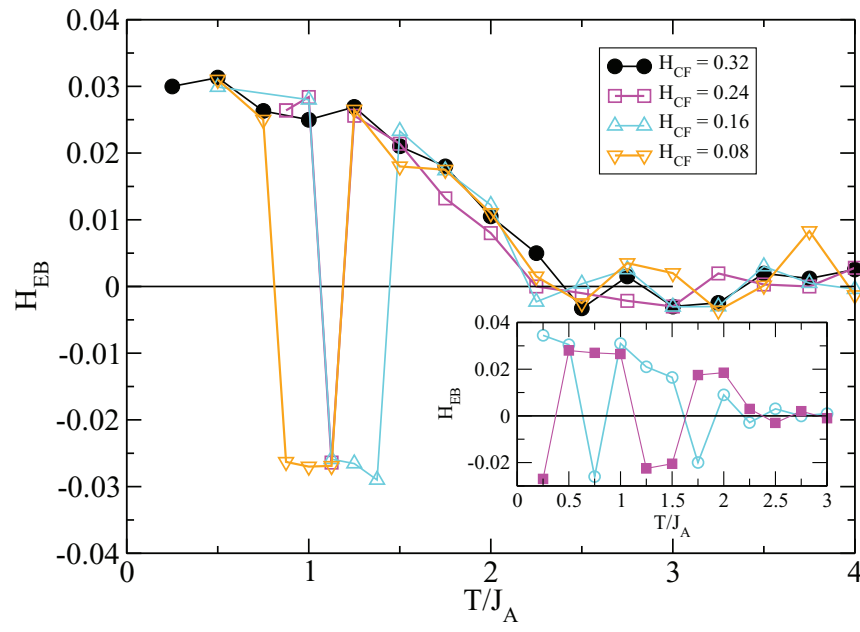

FIG. 2. (Color online) Bias field as function of the temperature. The AFM film includes 12 layers with disorder. Different symbols correspond to different cooling fields $L=40$. Inset: two samples in a reduced system size: $L=20$ and $H_{\mathrm{CF}}=0.08$.

Zeeman coupling of the AFM spins. This energy is restored during the field reversal producing a positive bias in the hysteresis loop. On the contrary, in the case of NEB, no DW is observed for positive field. In other words, the whole AFM slab (both the ordered and the disordered regions) exhibits a single Néel state without frustration. In this case, a DW forms for negative fields, giving rise to a negative bias of the hysteresis loop. It is worth noting that in our simulations, the DW formed in the disordered region is responsible for the shift in the magnetization.

Let us analyze the conditions for the formation of a domain wall under an applied field in the disordered region of the AFM. The energy per unit of area of an AFM disordered region of length $l_{w}$ under the applied field is ${ }^{32}$

$$
E=\int_{0}^{l_{w}}\left[\frac{J_{l}}{2}\left(\frac{d \theta}{d z}\right)^{2}-\frac{H_{\mathrm{CF}}}{2} \sin (\theta)\left(\frac{d \theta}{d z}\right)\right] d z .
$$

Assuming that randomness averages the effect of the anisotropy, we neglected it considering only the exchange interaction between layers $\left(J_{l}\right)$ and the coupling with the field. We will test this approximation later. Note that the field interacts through the gradient of the angle $\theta$ since this region is antiferromagnetically ordered, i.e., the magnetization in the direction of the field is $m(z)=\frac{1}{2} \sin (\theta)\left(\frac{d \theta}{d z}\right)$. Minimizing this energy, we obtain $\theta=\frac{\pi-\theta_{0}}{l_{w}} z+\theta_{0}$, where $\theta_{0}$ is a free parameter $\left(0<\theta_{0}<\pi\right)$. The total energy is

$$
E=\frac{J_{l}}{2}\left(\frac{\pi-\theta_{0}}{l_{w}}\right)^{2} l_{w}-\frac{H_{\mathrm{CF}}}{2}\left[1+\cos \left(\theta_{0}\right)\right],
$$

and a domain wall forms if $E<0$ implying $H_{\mathrm{CF}}>H^{*}$, where

$$
H^{*}=J_{l} \frac{\left(\pi-\theta_{0}\right)^{2}}{\left[1+\cos \left(\theta_{0}\right)\right] l_{w}} .
$$

The magnetization profile is

$$
m(z)=\frac{1}{2} \frac{\pi-\theta_{0}}{l_{w}} \sin \left(\frac{\pi-\theta_{0}}{l_{w}} z+\theta_{0}\right) .
$$




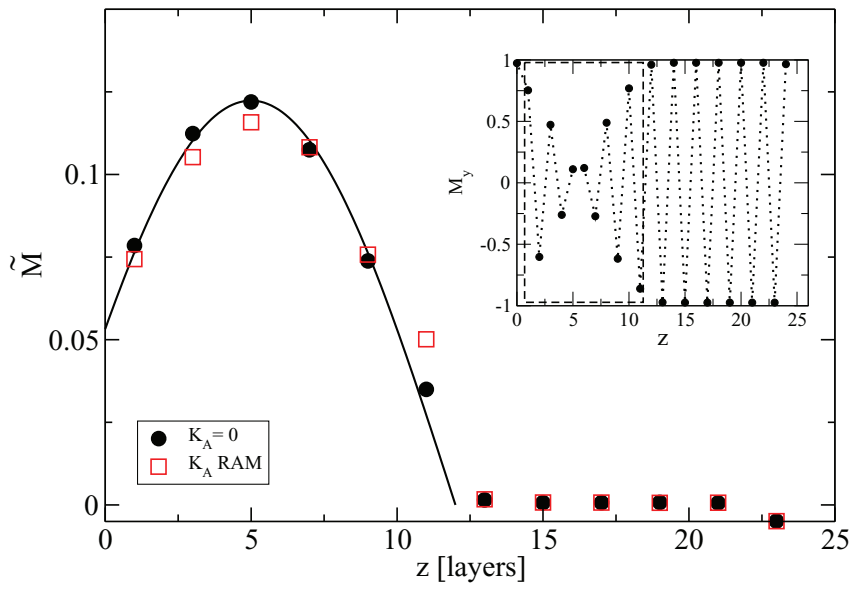

FIG. 3. (Color online) Net magnetization of the AFM $\tilde{M}(z)$ obtained through Monte Carlo simulations; RAM refers to random anisotropy. The solid line corresponds to Eq. (5) with $\theta_{0}=0.45$ and $l_{w}=11$. Inset: AFM magnetization profile; the dashed box indicates the disordered region.

We checked Eq. (5) simulating [Eq. (1)] a system containing a region without anisotropy. In Fig. 3, we plot the profile of the net magnetization $\tilde{M}(z)=\left[m_{y}(z)+m_{y}(z+1)\right] / 2$ pointing in the direction of the applied field. The continuous line is a fit of Eq. (5) with $\theta_{0}=0.45$, showing a good agreement with the Monte Carlo simulation. Moreover, when randomness is considered $\left(K_{A} \neq 0\right)$, the agreement is still good, verifying our previous assumption.

According to Eq. (4) there is a threshold for the appearance of a domain wall and therefore PEB. Using the parameters of the simulations (Fig. 3) and $J_{l}=4 J_{A}$, we obtain $H^{*}=$ $1.38 J_{A}(\sim 5.5 \mathrm{~T}) .{ }^{33}$ However, in simulations PEB is observed at cooling fields as low as $0.08 J_{A}(\sim 0.32 \mathrm{~T})$. To explain this discrepancy, one has to assume the domain wall forms at higher temperatures where $J_{l} \ll 4 J_{A}$. This is plausible since PEB is observed in our simulations even at temperatures close to $T_{N} \cdot{ }^{34}$

To analyze thermal effects in the DW formation mechanism, we consider a phenomenological model. Assuming that the spins at each AFM layer behave coherently, we associate an Ising spin $\sigma_{i}= \pm 1(i=1, \ldots, l+L)$, corresponding to the magnetization per unit area component in the direction of the applied field for the layer $i$. The magnetization per unit area of the FM slab is represented by $S=t_{F} \sigma_{0}$, where $t_{F}$ is the thickness of the FM slab and $\sigma_{0}= \pm 1$. The disordered interface is represented by the first $l$ layers $(i=1, \ldots, l)$. We assume that the anisotropy at the ordered region $i=$ $l+1, \ldots, l+L(L \gg l)$ is very strong, so that the Zeeman contribution of that region can be neglected. On the other hand, we assume the anisotropy at the disordered region can be neglected, compared with the corresponding Zeeman term. Then, the Hamiltonian for the effective model is given by

$$
\mathcal{H}=-J t_{F} \sigma_{0} \sigma_{1}+J_{\mathrm{AF}} \sum_{i=1}^{l+L-1} \sigma_{i} \sigma_{i+1}-H t_{F} \sigma_{0}-H \sum_{i=1}^{l} \sigma_{i}
$$

with $J>0$ and $J_{\mathrm{AF}}>0$. At zero temperature, a simple analysis of Eq. (6) shows the existence of a threshold $H^{*}=J_{\mathrm{AF}}$ such that a DW forms only when $H>H^{*}$, consistently with the previous micromagnetic calculation.

At finite temperature, a variational mean-field free energy can be easily derived ${ }^{35,36}$ in terms of the local average magnetizations $m_{i}=\left\langle\sigma_{i}\right\rangle \quad(i=0, \ldots, \infty)$. During FC, the ordered AFM slab $(i>l)$ takes a configuration that minimizes the whole free energy. Such a configuration remains fixed when the field is retired at low temperatures, while the disordered AFM region and the FM slab (i.e., those spins which interact with the field) are capable of accommodating a new minimum free-energy configuration. The analysis can be further simplified by assuming that the ordered AFM conforms to a Néel state with the local sublattice magnetization given by the Curie equation $m_{\mathrm{AF}}=\tanh \left[2 \beta J_{\mathrm{AF}} m_{\mathrm{AF}}\right]\left(\beta=1 / k_{B} T\right)$. We then have two different possibilities (let us assume for simplicity that $l$ is even): (i) $m_{l+1}=m_{\mathrm{AF}}$ (we are considering the positive root of the previous Curie equation and assuming $\left.T<T_{N}=2 J_{\mathrm{AF}} / k_{B}\right)$. In this case, there is no DW and $m_{F} \equiv t_{F}\left\langle m_{0}\right\rangle>0$ when $H=0$, thus corresponding to NEB. (ii) $m_{l+1}=-m_{\mathrm{AF}}$. There is a DW and $m_{F}<0$ when $H=$ 0 , thus corresponding to PEB. We numerically obtained the minimum free-energy solution for both possibilities and compared them for different values of $H$ and $T$. In Fig. 4 we show a typical example of both free energies when ${ }^{37} H<J_{\mathrm{AF}}$. We see that the minimum free-energy solution changes from NEB to PEB as the temperature increases. Conversely, for each temperature we have a minimum field $H^{*}(T)$ (see the lower inset of Fig. 4) such that PEB becomes the minimum free-energy state when $H>H^{*}(T)$, even for temperatures close to $T_{N}$. The upper inset of Fig. 4 shows the DW in the PEB case (compare with the inset of Fig. 3). A change in the sign of $H_{\mathrm{EB}}$ as a function of the temperature has been observed in disordered fluorides ${ }^{11,13-15}$ following the same trend we observed in the MF calculations. In particular, in the $\mathrm{Fe}_{x} \mathrm{Ni}_{1-x} \mathrm{~F}_{2} / \mathrm{Co}$ bilayer ${ }^{15}$ a domain wall at the interface has been reported, where $\mathrm{Fe}_{x} \mathrm{Ni}_{1-x} \mathrm{~F}_{2}$ is a random anisotropy antiferromagnet. Since the critical field $H^{*}$ depends on the amount

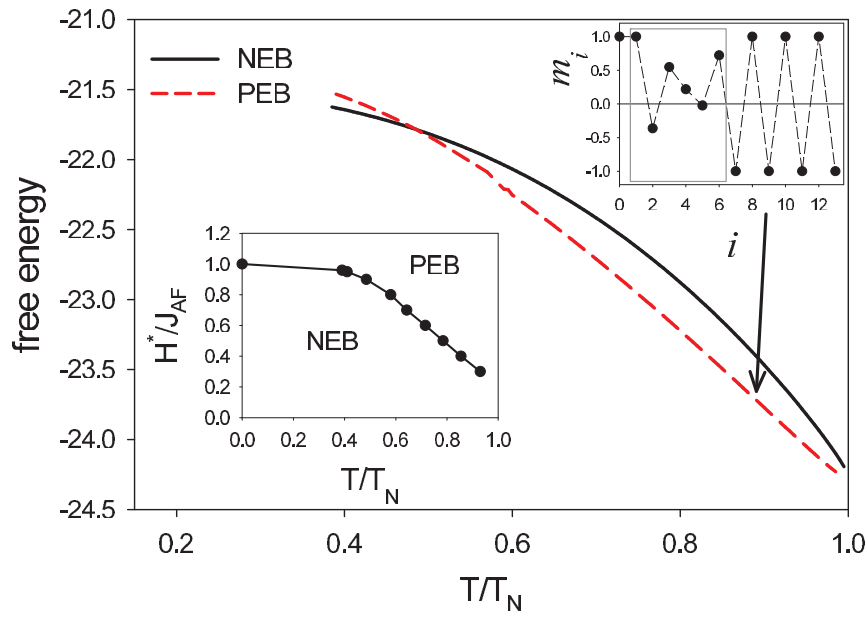

FIG. 4. (Color online) Mean-field free energy for Hamiltonian (6) as a function of the temperature for $H=0.9$ and $l=6$ corresponding to PEB (red dashed line) and NEB (continuous black line) AFM configurations. Upper inset: local magnetization at the PEB for $T=$ $0.85 T_{N}$. Lower inset: minimum cooling field for having PEB as a function of the temperature. 
of disordered layers, inhomogeneities at the interface can give rise to a distribution of $H^{*}$ as is observed in $\mathrm{FeF}_{2} / \mathrm{FM}^{38,39}$ Finally, the effect of the disorder of the anisotropy at the interface is similar to that considered in the spin glass model of exchange bias, ${ }^{40}$ since disorder reduces the anisotropy at the interface, but in our case the coupling of this region with the applied field turns out to be important to produce PEB.

Summarizing, the reduction in the anisotropy for a large enough amount of interfacial disorder can induce the formation of a domain wall in the cooling field process inducing a symmetry breaking in the antiferromagnet. The energy stored in this domain wall is released during the field reversion, resulting in $\mathrm{PEB}$. In this way, the $\mathrm{PEB}$ phenomenon can be explained as an exclusive result of interfacial disorder, without relying on ad hoc assumptions about the sign of the coupling between FM and AFM.

This work was partially supported by grants from CONICET and SeCyT Universidad Nacional de Córdoba (Argentina). *billoni@famaf.unc.edu.ar

${ }^{1}$ W. H. Meiklejohn and C. P. Bean, Phys. Rev. 102, 1413 (1956).

${ }^{2}$ B. Dieny, V. S. Speriosu, S. S. P. Parkin, B. A. Gurney, D. R. Wilhoit, and D. Mauri, Phys. Rev. B 43, 1297 (1991).

${ }^{3}$ A. Roy, R. Gupta, and A. Garg, Adv. Condens. Matter Phys. 2012, 926290 (2012).

${ }^{4}$ A. E. Berkowitz and T. Kentaro, J. Magn. Magn. Mater. 200, 552 (1999).

${ }^{5}$ J. Nogués, J. Sort, V. Langlais, V. Skumryev, S. Suriñach, J. S. Muñoz, and M. D. Baró, Phys. Rep. 422, 65 (2005).

${ }^{6}$ S. Giri, M. Patra, and S. Majumdar, J. Phys.: Condens. Matter 23, 073201 (2011).

${ }^{7}$ F. Radu, R. Abrudan, I. Radu, D. Schmitz, and H. Zabel, Nat. Commun. 3, 715 (2012).

${ }^{8}$ J. Nogués, D. Lederman, T. J. Moran, and I. K. Schuller, Phys. Rev. Lett. 76, 4624 (1996).

${ }^{9}$ J. Nogués, C. Leighton, and I. K. Schuller, Phys. Rev. B 61, 1315 (2000).

${ }^{10}$ K. Takano, R. H. Kodama, A. E. Berkowitz, W. Cao, and G. Thomas, Phys. Rev. Lett. 79, 1130 (1997).

${ }^{11}$ H. Shi, D. D. Lederman, N. R. Dilley, R. C. Black, J. Diedrichs, K. Jensen, and M. B. Simmonds, J. Appl. Phys. 93, 8600 (2003).

${ }^{12}$ J.-I. Hong, T. Leo, D. J. Smith, and A. E. Berkowitz, Phys. Rev. Lett. 96, 117204 (2006).

${ }^{13}$ M. Cheon, Z. Liu, and D. Lederman, J. Appl. Phys. 101, 09E503 (2007).

${ }^{14}$ M. Cheon, Z. Liu, and D. Lederman, Appl. Phys. Lett. 90, 012511 (2007).

${ }^{15}$ K. Munbodh, M. Cheon, D. Lederman, M. R. Fitzsimmons, and N. R. Dilley, Phys. Rev. B 84, 214434 (2011).

${ }^{16}$ C. Leighton, J. Nogués, H. Suhl, and I. K. Schuller, Phys. Rev. B 60, 12837 (1999).

${ }^{17}$ J. Nogués, T. J. Moran, D. Lederman, I. K. Schuller, and K. V. Rao, Phys. Rev. B 59, 6984 (1999).

${ }^{18}$ N. C. Koon, Phys. Rev. Lett. 78, 4865 (1997).

${ }^{19}$ T. C. Schulthess and W. H. Butler, Phys. Rev. Lett. 81, 4516 (1998).

${ }^{20}$ M. Kiwi, J. Mejía-López, R. D. Portugal, and R. Ramírez, Europhys. Lett. 48, 573 (1999).

${ }^{21}$ M. Kiwi, J. Mejía-López, R. D. Portugal, and R. Ramírez, Appl. Phys. Lett. 75, 3395 (1999).
${ }^{22}$ M. Kiwi, J. Mejía-López, R. D. Portugal, and R. Ramírez, Solid State Commun. 116, 315 (2000).

${ }^{23}$ U. Nowak, K. D. Usadel, J. Keller, P. Miltényi, B. Beschoten, and G. Güntherodt, Phys. Rev. B 66, 014430 (2002).

${ }^{24}$ Y. Hu and A. Du, Phys. Status Solidi B 248, 2932 (2011).

${ }^{25}$ O. V. Billoni, S. A. Cannas, and F. A. Tamarit, Phys. Rev. B 72, 104407 (2005).

${ }^{26}$ U. Nowak, A. Misra, and K. D. Usadel, J. Magn. Magn. Mater. 240, 243 (2002).

${ }^{27}$ We fixed the parameters to be $J_{F}=9.56 J, J_{A}=-J$, and $K_{F}=$ $-0.5 J$, where $J$ is an arbitrary parameter that sets the energy units. $J_{\mathrm{EB}}=0.5 \mathrm{~J}, \mathrm{~J}$ and $K_{A}=1.77 \mathrm{~J}$.

${ }^{28}$ O. V. Billoni, F. A. Tamarit, and S. A. Cannas, Physica B 384, 184 (2006).

${ }^{29}$ D. Lederman, R. Ramírez, and M. Kiwi, Phys. Rev. B 70, 184422 (2004).

${ }^{30}$ O. V. Billoni, S. A. Cannas, and F. A. Tamarit, J. Phys.: Condens. Matter 23, 386004 (2011).

${ }^{31}$ J.-V. Kim and R. L. Stamps, Appl. Phys. Lett. 79, 2785 (2001).

${ }^{32}$ See Supplemental Material at http://link.aps.org/supplemental/ 10.1103/PhysRevB.88.020405 for more details about these calculations.

${ }^{33}$ The molecular field of $\mathrm{FeF}_{2}$ is $8 J_{A} \sim 32 \mathrm{~T}$.

${ }^{34}$ The blocking temperatures in Fig. 2 are about 0.9 the Neél temperature.

${ }^{35}$ P. M. Chaikin and T. C. Lubensky, Principles of Condensed Matter Physics (Cambridge University Press, Cambridge, UK, 1995).

${ }^{36}$ See Supplemental Material at http://link.aps.org/supplemental/ 10.1103/PhysRevB.88.020405 for more details about the derivation of the free energy and the calculations described in what follows.

${ }^{37} \mathrm{We}$ used in the calculations $J_{\mathrm{AF}}=1, J=J_{\mathrm{AF}} / 2$, and $t_{F}=5$.

${ }^{38}$ O. Petracic, Z.-P. Li, I. V. Roshchin, M. Viret, R. Morales, X. Batlle, and I. K. Schuller, Appl. Phys. Lett. 87, 222509 (2005).

${ }^{39}$ I. V. Roshchin, O. Petracic, R. Morales, Z.-P. Li, X. Batlle, and I. K. Schuller, Europhys. Lett. 71, 297 (2005).

${ }^{40} \mathrm{~F}$. Radu and H. Zabel, Magnetic Heterostructures: Advances and Perspectives in Spinstructures and Spintransport, Springer Tracts in Modern Physics Vol. 227 (Springer-Verlag, Berlin, Heidelberg, 2008). 